\title{
Características clínicas de la neurocisticercosis en un hospital referencial del norte del Perú. 2016-2018
}

\section{Clinical characteristics of neurocysticercosis from a reference hospital of the northern of Peru. 2016-2018}

Benigno Ballón-Manrique1, Franco Ernesto León Jiménez² y James Joel Alcántara Vásquez

'Hospital Regional Lambayeque. Perú.

${ }^{2}$ Hospital Santa Rosa de Piura. Perú.

Los autores no presentan conflictos de interés.

Estudio financiado por los propios autores.

Recibido: 24 de enero de 2020 / Aceptado: 6 de octubre de 2020

\section{Resumen}

Introducción: La neurocisticercosis (NCC) es la parasitosis más común del sistema nervioso central, siendo una causa muy importante de epilepsia. Objetivo: Describir las características de pacientes con NCC atendidos en un hospital de alta complejidad de Lambayeque durante el período 2016-2018. Pacientes y Métodos: Se revisaron las historias clínicas de pacientes con diagnóstico de NCC y se recolectó su información en una ficha de datos. Resultados: 46 historias cumplieron criterios de inclusión; 23 correspondían a varones, la mediana de edad fue 46,5 años (RIC: $26,5-63$ ), el paciente más joven tuvo 7 años, el más longevo 85 años; 30 procedían de la región Lambayeque. Epilepsia se presentó en 24 pacientes, hipertensión endocraneal en 10 , síndrome psíquico en dos, déficit neurológico focal en 1, síndrome visual en 1 , un paciente fue asintomático. Siete pacientes tuvieron epilepsia y otro síndrome simultáneamente. En las neuroimágenes, las calcificaciones cerebrales fueron las lesiones más comunes; 9 tuvieron quistes sub-aracnoideos. En 20 pacientes se efectuó serología por western blot, siendo positiva en 11; 38 fueron clasificados como NCC definitiva y 8 probable. Recibieron solamente tratamiento sintomático 18 pacientes, 27 tratamiento antiparasitario y 6 adicionalmente tratamiento neuroquirúrgico. Falleció un paciente. Conclusiones: La sintomatología y los hallazgos de neuroimágenes fueron proteiformes y la mortalidad fue baja.

Palabras clave: neurocisticercosis; manifestaciones clínicas; epilepsia.

\section{Abstract}

Backgound: Neurocysticercosis (NCC) is the most common parasitosis of the central nervous system, and a very important cause of epilepsy. Aim: To describe the clinical features of patients with NCC attending a high level hospital from Lambayeque during: 2016-2018. Methods: The medical records of patients with NCC were reviewed, and their information was collected on a data sheet. Results: 46 stories met the inclusion criteria; 23 patients were male, the median age was 46.5 years (IQR: $26.5-63$ ), the youngest patient was 7 years old, and the longest 85 . Thirty patients were from Lambayeque. Epilepsy occurred in 24 patients, intracranial hypertension in 10, psychic syndrome in 2 and focal neurological deficit and visual syndrome in 1 ; there was one asymptomatic patient and seven had epilepsy and another syndrome. In neuroimaging, cerebral calcifications were the most common lesions; 9 patients had subarachnoid cysts. Serology (western blot) was performed in 20 patients being positive in 11; 38 were definitive NCC and 8 probable. Eighteen patients received only symptomatic treatment, 27 antiparasitic treatment and 6, additionally neurosurgical treatment. Only one patient died. Conclusions: The symptoms and neuroimaging findings were proteiform and the mortality found was low.

Keywords: neurocysticercosis; signs and symptoms; epilepsy. 
una ficha con datos epidemiológicos, clínicos, hallazgos de neuroimágenes, resultados de exámenes serológicos, tipo de diagnóstico y tratamiento recibido por el paciente. Las características clínicas se agruparon en síndromes clínicos; los pacientes que presentaban cefalea como síntoma aislado, se incluyeron dentro del síndrome de hipertensión endocraneal. En las neuroimágenes, se definió NCC múltiple si existían más de 50 lesiones quísticas y NCC gigante si el quiste medía más de $3 \mathrm{~cm}$.

ra luego localizarse en distintos órganos; generalme ocasiona síntomas si se localiza en el SNC o en el ojo ${ }^{2}$. En el cerebro el parásito puede permanecer inalterado como una vesícula o quiste o puede entrar en contacto con el sistema inmunológico y pasar por las fases coloidal, granulomatosa y calcificada ${ }^{3}$. La localización dentro del SNC puede ser: intra-parenquimatosa, intra-ventricular, subaracnoidea e intra-medular ${ }^{1}$. La sintomatología depende de la localización, el estadio y el número de parásitos. El síndrome clínico más frecuente es la epilepsia; en zonas endémicas, entre 30 y $50 \%$ de pacientes con epilepsia corresponden a casos de $\mathrm{NCC}^{4}$.

Se han propuesto diversos criterios diagnósticos para NCC, basados en las manifestaciones clínicas, los estudios de neuroimágenes y los estudios inmunológicos en suero ${ }^{5}$.

El tratamiento es variable, pues depende de la localización, número y estadio de los parásitos; puede ser sólo sintomático o requerir el uso de antiparasitarios y, en algunos casos, pueden realizarse procedimientos neuroquirúrgicos como la exéresis de quistes o la colocación de sistemas de derivación ventricular ${ }^{1}$.

No hemos encontrado un estudio en nuestro país que describa integralmente las características epidemiológicas, clínicas y los hallazgos de los resultados de exámenes auxiliares de pacientes con esta enfermedad. El presente trabajo buscar describir estas características en pacientes atendidos en un hospital referencial del norte del Perú, durante un período de tres años.

\section{Pacientes y Métodos}

El Hospital Regional Lambayeque (HLR) es un recinto referencial de alta complejidad, ubicado en la región costera al noroeste del Perú. Atiende, principalmente, a población procedente de la región de Lambayeque y de las regiones vecinas de Piura y Cajamarca.

Para la recolección de datos, se recopilaron las historias clínicas registradas con el CIE10 B69.0 correspondiente a $N C C$. Se revisaron las historias clínicas de pacientes atendidos durante el período comprendido entre los años 2016 y 2018 . Se incluyeron pacientes con historia clínica completa, con estudios de neuroimágenes (tomografía de cráneo y/o resonancia magnética [RM] de encéfalo) y que cumplían los criterios diagnósticos actualmente recomendados para NCC. Se recolectó la información en

\section{Resultados}

Del total de 75 historias; sólo 46 cumplían con los criterios de inclusión; 23 fueron varones, mediana de edad: 46,5 años (RIC: 26,5-63), el paciente más joven tuvo 7 años, y el más longevo 85 años. Treinta procedían de la región Lambayeque, ocho de la región Cajamarca, siete de la región Piura y uno de Lima.

El síndrome epiléptico se presentó en 24 pacientes, el síndrome de hipertensión endocraneal en 10, síndrome psíquico en dos y los síndromes de déficit neurológico focal y visual en uno; un paciente fue asintomático. Siete pacientes tuvieron un síndrome epiléptico asociado simultáneamente a otro síndrome. El paciente que fue asintomático ingresó a emergencia por traumatismo craneal, e incidentalmente se encontró quistes viables en la tomografía.

A 27 pacientes se les realizó tomografía de cráneo y RM de encéfalo, a 15 tomografía y a 4 sólo RM. Los hallazgos de neuroimágenes se describen en la Tabla 1; 10 tuvieron más de un estadio y/o localización del parásito; nueve cursaron con quistes sub-aracnoideos.

Sólo a 20/46 se les tomó western blot para NCC: fueron positivos 11 y negativos 9 .

\begin{tabular}{ll}
$\begin{array}{l}\text { Tabla 1. Hallazgos de neuroimágenes de los pacientes con } \\
\text { neurocisticercosis (NCC). Hospital Regional Lambayeque } \\
\text { 2016-2018 (n: 46) }\end{array}$ & $\mathbf{n}$ \\
\hline Tipo de lesión y localización & 10 \\
\hline Calcificaciones y otro tipo de lesiones* & 9 \\
\hline Sub-aracnoidea & 8 \\
\hline Calcificaciones parenquimatosas & 7 \\
\hline Granulomas parenquimatosos & 4 \\
\hline Quiste parenquimatoso & 3 \\
\hline Quiste intra-ventricular & 3 \\
\hline NCC múltiple & 2 \\
\hline Quiste gigante parenquimatoso & \\
\hline *Quiste o granuloma. & \\
\hline
\end{tabular}


Según los criterios diagnósticos de NNC actualmente propuestos, 38 pacientes tuvieron diagnóstico de $\mathrm{NCC}$ definitiva y 8 pacientes de NCC probable. Sólo un paciente tuvo hallazgo de NCC por microscopia luego de la exéresis quirúrgica de un quiste gigante.

En 18 pacientes se administró tratamiento sintomático solamente, siendo el más común el uso de antiepilépticos, 27 requirieron tratamiento antiparasitario con albendazol y 6 requirieron adicionalmente tratamiento neuroquirúrgico, principalmente con la colocación de sistemas de derivación ventrículo-peritoneal. Uno de los pacientes falleció, este paciente presentaba NCC sub-aracanoidea.

\section{Discusión y conclusiones}

Describimos las principales características epidemiológicas, manifestaciones clínicas, y resultados de exámenes auxiliares en pacientes con NCC atendidos en el HLR.

La mayoría de los pacientes han sido adultos jóvenes, con una mediana de 46,5 años, hallazgo similar a otros reportes $^{1,6}$; la NCC es inusual en los extremos de la vida. Se especula que existen factores inmunológicos relacionados con la edad y el sexo que afectan el desarrollo del parásito en los diversos grupos etarios. De igual manera a lo descrito en otras instituciones, no se halló una frecuencia diferente según sexo ${ }^{6}$.

La mayoría de los pacientes provinieron de la región Lambayeque, pero también hubo un número importante de las regiones vecinas de Piura y Cajamarca; nuestro hospital es un centro referencial que cuenta con equipos de tomografía y RM, los que no están disponibles en otras regiones. Este factor hace que pacientes de regiones vecinas acudan al HRL para su diagnóstico y tratamiento.

El HRL se ubica en la costa norte del Perú y atiende a pacientes de regiones vecinas de la sierra norte. Se ha reportado que la NCC es endémica en nuestro país; en las regiones de costa norte, sierra y selva alta, la seroprevalencia alcanza a $10-20 \%$ en la población general ${ }^{7}$.

Al igual que otros reportes ${ }^{6,8}$, la epilepsia fue el síndrome clínico más frecuente. También se hallaron otros síndromes clínicos reportados como la hipertensión endocraneal, déficit neurológico focal o síndromes psíquicos como deterioro cognitivo o psicosis ${ }^{1}$. Notamos con interés que un grupo de pacientes presentaban simultáneamente más de un síndrome clínico, siendo la asociación de síndrome epiléptico con hipertensión endocraneal la forma más común.

Tanto la tomografía como la RM son útiles para el diagnóstico ${ }^{9}$. A la mayoría de los pacientes se les practicó ambos estudios; la indicación de los mismos era de acuerdo al criterio médico y a la disponibilidad del estudio. Los hallazgos de neuroimágenes mostraron de manera similar a anteriores reportes, que la forma calcificada fue la más común ${ }^{10} ;$ los pacientes también presentaron otras formas descritas como quistes intra-parenquimatosos, quistes gigantes, granulomas, quistes intra-ventriculares y quistes sub-aracnoideos. Este último se presentó en un importante número de casos. Ello pone manifiesto la necesidad de un examen neurológico prolijo y la importancia del uso de la RM.

Un grupo de pacientes presentaba simultáneamente quistes asociados a otras lesiones como granulomas o calcificaciones cerebrales. No hemos encontrado información de otras investigaciones que reporte cuantitativamente la presentación simultánea de lesiones de NCC. Estos hallazgos nos indican que el parásito puede localizarse simultáneamente en distintas zonas del SNC, y que un paciente puede tener múltiples lesiones en diferentes estadios. Probablemente ello se deba a que el sistema inmunológico sólo ataca a algunos de ellos y sólo algunos quistes son eliminados. Por ello, cada caso debe ser individualizado y tratado de acuerdo al tipo de lesiones halladas, combinando tratamientos médico y quirúrgico. También comentaremos que la presencia de más de un estadio del parásito facilita el proceso de diagnóstico, sobre todo en formas inespecíficas como los granulomas, calcificaciones o la hidrocefalia.

En el HRL, según criterio médico, se solicita western blot, priorizando aquellos pacientes cuyos estudios de neuroimágenes no son concluyentes para el diagnóstico (por ejemplo: lesiones granulomatosas intra-parenquimatosas); 9/20 tuvieron western blot negativo, cifra coincidente con otros reportes donde hasta $60 \%$ de pacientes con lesiones granulomatosas, calcificadas o quistes únicos pueden tener un resultado negativo ${ }^{11}$. Debemos comentar también que a ningún paciente sistemáticamente se le solicitó estudios en heces que, aunque tienen muy baja sensibilidad, se recomiendan para búsqueda de la $T$. solium (huevos o proglótidas) para información epidemiológica.

De acuerdo a los criterios diagnósticos propuestos, 38 pacientes tuvieron diagnóstico definitivo y ocho un diagnóstico probable. Sólo uno de nuestros pacientes tuvo hallazgo de NCC por microscopia luego de la exéresis quirúrgica de un quiste gigante; en el resto de pacientes, los hallazgos de neuroimágenes fueron la base para hacer el diagnóstico de NCC.

A todos los pacientes se les indicó tratamiento sintomático; se utilizó solamente tratamiento sintomático en pacientes que tenían formas calcificadas, granulomas o NCC masiva. Adicionalmente a este tratamiento, los pacientes con lesiones quísticas intra-parenquimatosas y NCC sub-aracnoidea recibieron tratamiento antiparasitario (en el HRL sólo tenemos disponible el antiparasitario albendazol). Seis pacientes requirieron, adicionalmente, tratamiento neuroquirúrgico.

Es conocido que la forma sub-aracnoidea de NCC tiene un peor pronóstico ${ }^{12}$, nueve de los pacientes tuvieron la 
forma sub-aracnoidea y uno de ellos falleció luego de la colocación de un sistema de derivación ventrículoperitoneal.

Entre las limitaciones del estudio se encuentran los sesgos inherentes a todo estudio retrospectivo, la falta de información epidemiológica de saneamiento de la vi- vienda, ocupación del paciente y hábitos de sus contactos.

En suma, describimos una serie de pacientes con NCC atendidos en un hospital referencial, con sus características epidemiológicas, clínicas y de exámenes auxiliares. Recomendamos realizar estudios prospectivos multicéntricos y por períodos de observación más prolongados.

\section{Referencias bibliográficas}

1. García H, Nash T, Brutto O. Clinical symptoms diagnosis and treatment of neurocysticercosis. Lancet Neurol 2014; 13: 1202-15. doi: $10.1016 /$ S1474-4422(14)70094-8.

2. García H. Brutto O, Nash T, White AC Jr, Tsang V C W, Gilman R H. New concepts in the diagnosis and managament of neurocysticercosis (Taenia solium). Am J Trop Med Hyg 2005; 72 (1): 3-9. PMID: 15728858.

3. Takayanagui OM, Clinton White Jr A, Botero D, et al. Current consensus guidelines for treatment of neurocysticercosis. Clin Microbiol Rev 2002; 15 (4): 747-6. doi: 10.1128/cmr.15.4.747-756.2002.

4. Villaran M V, Montano S M, Gonzalvez G, Moyano L M, Chero J C, Rodríguez S, et al. Epilepsy and neurocysticercosis: an incidence study in a Peruvian rural population. Neuroepidemiology 2009; 33 (1): 25-31. doi: 10.1159/000210019.
5. Del Brutto O H, Nash T E, White A C Jr, Rajshekhar V, Wilkins P P, Singh $\mathrm{G}$, et al. Revised diagnostic criteria for neurocysticercosis. J Neurol Sci. 2017; 372: 202-10. doi: 10.1016/j.jns.2016.11.045.

6. Velasquez-Pérez L, Cruz-Olano J L, Juarez Olivera S G. Neurocisticercosis: algunos aspectos epidemiológicos de los casos diagnosticados en el Instituto Nacional de Neurología y Neurocirugía 1995-2001. Rev Mex Neuroci 2004; 5 (5): 426-33. http:// previous.revmexneurociencia.com/wp-content/ uploads/2014/07/Nm0045-05.pdf.

7. García $\mathrm{H} \mathrm{H}$, Gonzales A E, Rodríguez $\mathrm{S}$, Gonzalves G, Llanos-Zavalaga F, Tsang V C, et al, Grupo de trabajo en cisticercosis Perú. Epidemiology and control of cysticercosis in Peru. Rev Per Med Exp Salud Pública. 2010; 27 (4): 592-7. http://www.scielo.org.pe/pdf/ rins/v27n4/a16v27n4.pdf.

8. Carabin H, Ndimubanzi P C, Budke C M, Nguyen H, Qian Y, Cowan L D, et al.
Clinical manifestations associated with neurocysticercosis: a systematic review. Plos Negl Trop Dis. 2011; 5 (5): 31152. doi: 10.1371/journal.pntd.0001152.

9. Lerner A, Shiroishi M S, Zee C S, Law $\mathrm{M}$, Go J L. Imaging of neurocysticercosis. Neuroimaging Clin N Am 2012; 22 (4): 659-76. doi: 10.1016/j.nic.2012.05.004.

10. Nash TE, Del Brutto OH, Butman JA, Corona T, Delgado-Escueta A, Duron RM, et al. Calcific neurocysticercosis and epileptogenesis. Neurology 2004 (8); 62(11): 1934-8. doi: 10.1212/01.wnl.0000129481.12067.06.

11. Rodríguez S, Wilkins P, Dorny P. Inmunological and molecular diagnosis of cysticercosis. Pathog Glob Health 2012; 106 : 286-9. doi: 10.1179/2047773212Y.0000000048.

12. Fleury A, Carrillo-Mezo R, Flisser A, Sciutto E, Corona T. Subarachnoid basal neurocysitcercosis: a focus on the most severe form of the disease. Expert Rev Anti Infect Ther. 2011; 9: 123-33. doi: 10.1586/eri.10.150. 